\title{
RADON-NIKODYM DERIVATIVES FOR BANACH LATTICE-VALUED MEASURES
}

\author{
EP DE JONGE ${ }^{1}$
}

\begin{abstract}
Let $(\Delta, \Gamma, \mu)$ be a measure space such that $0<\mu(\Delta)<\infty$ and such that $\Gamma$ has no $\mu$-atoms. Furthermore, let $E$ be a Dedekind complete Banach lattice. By $M(\mu, E)$ we denote the set of all $E$-valued set functions $\nu$ on $\Gamma$ satisfying

(i) $\nu$ is additive,

(ii) $\nu$ is order bounded and of bounded variation,

(iii) $\nu$ is $\mu$-absolutely continuous (with respect to the norm topology on $E$ ),

(iv) $\nu^{+}$and $\nu^{-}$satisfy

$$
\inf \left\{\sup \left\{v^{+/-}(A): \mu(A)<\varepsilon\right\}: \varepsilon>0\right\}=0 .
$$

By $L_{1}(\mu, E)$ we denote the set of $E$-valued Bochner integrable functions on $\Delta$. It is shown that under the canonical map

$$
f \mapsto \nu_{f}
$$

(where $\left.\nu_{f}(A)=\int f \chi_{1} d \mu, A \in \Gamma\right), L_{1}(\mu, E)$ is a Riesz subspace of the Dedekind complete Riesz space $M(\mu, E)$. Furthermore, the following theorems are proved.

THEOREM. Equivalent are
(a) $l_{\infty}$ is not isomorphic to a closed sublattice of $E$.
(b) $L_{1}(\mu, E)$ is isomorphic to an ideal in $M(\mu, E)$.
THEOREM. Equivalent are
(a) $c_{0}$ is not isomorphic to a closed sublattice of $E$.
(b) $L_{1}(\mu, E)$ is isomorphic to $M(\mu, E)$.

1. Introduction. It is well known that $L_{1}$ does not have the Radon-Nikodym Property (RNP). However, we shall show in Theorem 3.3 below that one can easily describe all $L_{1}$-valued measures that have a $R N$-derivative. In fact, we do this for all Banach lattices $E$ not containing $c_{0}$. Also, we show that if a Banach lattice has order continuous norm, then it can be decided for many vector measures into $E$ whether they have a RN-derivative or not. So this will not be a paper on the RNP for Banach lattices (the reader interested in that subject is also referred to [4] and [6]).

Let $(\Omega, \Sigma, \mu)$ always be an atomless measure space such that $0<\mu(\Omega)<\infty$ and let $E$ be a Dedekind complete Banach lattice with norm $\|\cdot\|$. Our notations and terminology will always follow [2] and [7] with the exception that the variation of a vector measure $\nu$ is denoted by $\|\nu\|$, thus leaving the notation $|\nu|$ for the absolute value of $\nu$. Crucial in our results will be the notion $\nu \lll \mu$ (Definition 2.1) below.

Received by the editors April 10, 1980.

1980 Mathematics Subject Classification. Primary 28B05; Secondary 46G10.

Key words and phrases. Radon-Nikodym derivatives, Bochner integral, Banach lattices.

'The work on this paper is supported by the Netherlands Organisation for Advancement of Pure Research (Z.W.O.). 
We note already that this notion has an obvious extension to the non-Dedekind complete case and that Theorem 3.1, which states that if $\nu$ has an RN-derivative in $L_{1}(\mu, E)$, then $\nu$ must satisfy the conditions

(i) $|\nu|$ exists and $|\nu| \lll \mu$,

(ii) $|\nu|$ is $\sigma$-additive, $\mu$-absolutely continuous and of bounded variation, is still true in the general case. Here $L_{1}(\mu, E)$ is the Banach space consisting of all $E$-valued Bochner integrable functions on $\Omega$. We note that $L_{1}(\mu, E)$ is in a canonical way a Banach lattice. By $B_{\infty}(\mu, E)$ we shall denote the ideal in $L_{1}(\mu, E)$ generated by the step functions. Note that $B_{\infty}(\mu, E)$ is a norm dense ideal which is generally strictly included in the ideal $L_{\infty}(\mu, E)$.

In $\$ 2$, we study $E$-valued measures on $\Sigma$, using without reference some results of [3].

In $\$ 3$ we answer the following questions.

1.1. For what Banach lattices do we have that every $\nu$ satisfying (i) and (ii) above has a RN-derivative?

1.2. For what Banach lattices do we have that the vector measures with a RN-derivative form an ideal?

We shall show that 1.1 is true if and only if $E$ does not contain $c_{0}$ and that 1.2 is true if and only if $E$ has order continuous norm. In the second case we are still able to describe precisely the measure coming from elements of $B_{\infty}(\mu, E)$.

2. Banach lattice-valued measures. From now on let $(\Omega, \Sigma, \mu)$ be an atomless measure space such that $0<\mu(\Omega)<\infty$ and let $E$ be a Dedekind complete Banach lattice. By $m(\Sigma, E)$ we shall denote the linear space consisting of all $E$-valued vector measures on $\Sigma$ (i.e., all $E$-valued finitely additive set functions on $\Sigma$ ). In this section we introduce and investigate several subspaces of $m(\Sigma, E)$. First, we denote by $\operatorname{BCA}(\mu, E)$ the Banach space of all $\nu \in m(\Sigma, E)$ which are $\sigma$-additive, $\mu$ absolutely continuous and of bounded variation. The norm in $\operatorname{BCA}(\mu, E)$ is given by

$$
\|\nu\|=\|\nu\|(\Omega)
$$

for all $\nu \in \operatorname{BCA}(\mu, E)$.

As usual we call a $\nu \in m(\Sigma, E)$ order bounded if the set $\{\nu(A): A \in \Sigma\}$ is order bounded in $E$. The collection of all order bounded elements in $m(\Sigma, E)$ is denoted by $\mathrm{ob}(\Sigma, E)$. Since $E$ is Dedekind complete it follows that $\mathrm{ob}(\Sigma, E)$ is in a canonical way a Dedekind complete Riesz space as well. If $\nu \in \mathrm{ob}(\Sigma, E)$, then $|\nu|$ will denote the absolute value of $\nu$ in the Riesz space ob $(\Sigma, E)$. We shall now introduce a notion that will play a key role in our investigations.

Definition 2.1. Let $\nu \in \mathrm{ob}(\Sigma, E)$. We shall write $\nu \lll \mu$ whenever

$$
\inf \left\{\sup \{|\nu|(A): \mu(A)<\varepsilon\}: \varepsilon>0 \text { in } \mathbf{R}^{+}\right\}=0 .
$$

Furthermore, we set

$$
\mathrm{ca}_{1}(\mu, E)=\{\nu \in \mathrm{ob}(\Sigma, E): \nu \lll \mu\} .
$$

In fact the notion $\nu \lll \mu$ is a kind of "uniform absolute continuity" based on the ordering in $E$. Observe already that it is obvious that $\mathrm{ca}_{1}(\mu, E)$ is an ideal in 
$\operatorname{ob}(\Sigma, E)$. Later on we shall see that it is even a band. Next, we introduce another set of measures, viz.

$$
\mathrm{ca}_{\infty}(\mu, E)=\left\{\nu \in \mathrm{ob}(\Sigma, E): \exists e_{\nu} \in E^{+} \text {such that }|\nu|(A)<\mu(A) e_{\nu} \forall A \in \Sigma\right\} .
$$

Again it is obvious that $\mathrm{ca}_{\infty}(\mu, E)$ is an ideal in ob( $(\Sigma, E)$ and clearly

$$
\mathrm{ca}_{\infty}(\mu, E) \subset \mathrm{ca}_{1}(\mu, E) \text {. }
$$

Finally, we introduce

$$
M(\mu, E)=\left\{\nu \in \mathrm{ob}(\Sigma, E):|\nu| \in \operatorname{BCA}(\mu, E) \cap \mathrm{ca}_{1}(\mu, E)\right\} .
$$

Again it is clear that $M(\mu, E)$ is an ideal in $\operatorname{ob}(\Sigma, E)$. We note that if $\nu \in \operatorname{ob}(\Sigma, E)$ is such that $|\nu| \in \operatorname{BCA}(\mu, E)$, then $\nu \in \operatorname{BCA}(\mu, E)$ but the converse does not have to be true. The reason for considering the above classes will become clear in the next section, but first we derive some properties of the above introduced classes of measures.

THEOREM 2.2. (i) $\mathrm{ca}_{\infty}(\mu, E) \subset M(\mu, E)$.

(ii) Let $\nu \in \operatorname{ob}(\Sigma, E)^{+}$be given. Then the followiing are equivalent.

(a) $\exists \nu_{n} \in \mathrm{ca}_{\infty}(\mu, E)^{+}(n \in N)$ such that $\nu_{n} \uparrow \nu$ (order convergence).

(b) $\exists \nu_{\tau} \in \mathrm{ca}_{1}(\mu, E)^{+}(\tau \in T)$ such that $\nu_{\tau} \uparrow \nu$.

(c) $\nu \in \mathrm{ca}_{1}(\mu, E)^{+}$.

(iii) The set $\mathrm{ca}_{1}(\mu, E)$ is the band as well as the $\sigma$-ideal in $\mathrm{ob}(\Sigma, E)$ generated by $\mathrm{ca}_{\infty}(\mu, E)$.

Proof. (i) is obvious and (iii) follows from (ii). To prove the equivalences of (ii) note that $(\mathrm{a}) \Rightarrow(\mathrm{b})$ is clear since $\mathrm{ca}_{\infty}(\mu, E) \subset \mathrm{ca}_{1}(\mu, E)$. To show (b) $\Rightarrow$ (c), let $\nu_{\tau} \in \mathrm{ca}_{1}(\mu, E)^{+}$be such that $\nu_{\tau} \uparrow \nu(\tau \in T)$. For $\tau \in T$, set $e_{\tau}=\nu(\Omega)-\nu_{\tau}(\Omega)$. Then $e_{\tau} \downarrow 0$. Next, for $\tau \in T$ and $A \in \Sigma$ we have

$$
0 \leqslant \nu(A)=\nu_{\tau}(A)+\nu(A)-\nu_{\tau}(A)<\nu_{\tau}(A)+e_{\tau} .
$$

Hence

$$
0<\inf \{\sup \{\nu(A): \mu(A)<\varepsilon\}: \varepsilon>0\}<e_{\tau}
$$

for all $\tau \in T$ since $\nu_{\tau} \lll \mu$. This shows that $\nu \lll \mu$. Finally assume that $\nu \lll \mu$. Let $e=\nu(\Omega)$ and for $n \in N$, set $\nu_{n}^{1}(A)=n \mu(A) e$ for all $A \in \Sigma$. Clearly $\nu_{n}^{1} \in$ $\mathrm{ca}_{\infty}(\mu, E)^{+}(n \in \mathrm{N})$. Setting $\nu_{n}=\nu \wedge \nu_{n}^{1}$ it follows that $\nu_{n} \in \mathrm{ca}_{\infty}(\mu, E)^{+}(n \in \mathrm{N})$ and that

$$
0<\nu_{1}<\nu_{2}<\cdots<\nu .
$$

Thus it remains to show that $\nu_{n}(\Omega) \uparrow \nu(\Omega)$. To this end, let $\varepsilon>0$ (in R) be given and set

$$
e(\varepsilon)=\sup \{\nu(A): \mu(A)<\varepsilon\} .
$$

Next, let $n \in \mathbf{N}$. Then we have

$$
\begin{aligned}
\nu(\Omega)-\nu_{n}(\Omega) & =e-\inf \left\{\nu\left(B^{c}\right)+\nu_{n}^{1}(B): B \in \Sigma\right\}=-\inf \{n \mu(B) e-\nu(B): B \in \Sigma\} \\
& =\sup \{\nu(B)-n \mu(B) e: \mu(B)>\varepsilon\} \vee \sup \{\nu(B)-n \mu(B) e: \mu(B)<\varepsilon\} \\
& <(e-n \varepsilon e) \vee e(\varepsilon) .
\end{aligned}
$$


Thus

$$
0 \leqslant \bigwedge_{n=1}^{\infty}\left(\nu(\Omega)-\nu_{n}(\Omega)\right)<e(\varepsilon) .
$$

Since $e(\varepsilon) \downarrow 0$ as $\varepsilon \downarrow 0$ (in view of $\nu \lll \mu)$, it follows that $\nu_{n}(\Omega) \uparrow \nu(\Omega)$ has to hold, which proves $(c) \Rightarrow(a)$.

3. The existence of Radon-Nikodym derivatives. Let $(\Omega, \Sigma, \mu)$ and $E$ be as in the preceding section. Given $f \in L_{1}(\mu, E)$, set

$$
\nu_{f}(A)=\int f \chi_{A} d \mu
$$

for all $A \in \Sigma$. It is clear that $\nu_{f} \in m(\Sigma, E)$. Defining $J: L_{1}(\mu, E) \rightarrow m(\Sigma, E)$ by setting $J(f)=\nu_{f}$ for all $f \in L_{1}(\mu, E)$, it follows that $J$ is linear. Moreover, it is well known that $J$ is a linear isometry of $L_{1}(\mu, E)$ into $\operatorname{BCA}(\mu, E)$. Now observe that $J$ maps $L_{1}(\mu, E)$ also into $\mathrm{ob}(\Sigma, E)$. Furthermore, by first considering step functions and then using the continuity of $J$ into $\operatorname{BCA}(\mu, E)$, it follows that $J$ is a Riesz homomorphism from $L_{1}(\mu, E)$ into $\mathrm{ob}(\Sigma, E)$. Much more can be said.

THEOREM 3.1. (i) The restriction of $J$ to $B_{\infty}(\mu, E)$ is Riesz homomorphism into $\mathrm{ca}_{\infty}(\mu, E)$.

(ii) $J$ is a Riesz homomorphism of $L_{1}(\mu, E)$ into $M(\mu, E)$.

Proof. (i) Let $f \in B_{\infty}(\mu, E)$. Then there exists an $e \in E^{+}$such that $|f|<e \chi_{\Omega}$. Setting $\nu(A)=\mu(A) e$ for all $A \in \Sigma$, it follows that $\nu \in \mathrm{ca}_{\infty}(\mu, E)^{+}$and that $|J(f)| \leqslant \nu$, so $J$ maps $B_{\infty}(\mu, E)$ into $\mathrm{ca}_{\infty}(\mu, E)$. Clearly it is a Riesz homomorphism.

(ii) Let $f \in L_{1}(\mu, E)^{+}$be given. Since $B_{\infty}(\mu, E)$ is a norm dense ideal, it follows that there exist $g_{1}, g_{2}, \ldots$ in $B_{\infty}(\mu, E)^{+}$such that $g_{n} \uparrow f$ and such that $\left\|g_{n}-f\right\|_{1} \rightarrow$ 0 . Hence $\left\|J\left(g_{n}\right)-J(f)\right\| \rightarrow 0$ in $\operatorname{BCA}(\mu, E)$, so it follows that $J\left(g_{n}\right) \uparrow J(f)$ in $\operatorname{ob}(\Sigma, E)$. By part (i) we know that $J\left(g_{n}\right) \in \mathrm{ca}_{\infty}(\mu, E)^{+}$for all $n$, so Theorem 2.2 shows that $J(f) \in \mathrm{ca}_{1}(\mu, E)$. This shows that $J$ is a Riesz homomorphism of $L_{1}(\mu, E)$ into $M(\mu, E)$.

In view of the above result one is tempted to ask for what Banach lattices $E$ the equalities $J\left(L_{1}(\mu, E)\right)=M(\mu, E)$ or $J\left(B_{\infty}(\mu, E)\right)=\mathrm{ca}_{\infty}(\mu, E)$ hold. We shall show that it is not hard to present these classes of Banach lattices.

THEOREM 3.2. The following are equivalent.

(a) $E$ has order continuous norm.

(b) $J\left(B_{\infty}(\mu, E)\right)=\mathrm{ca}_{\infty}(\mu, E)$.

(c) $J\left(L_{1}(\mu, E)\right)$ is an ideal in $M(\mu, E)$.

(d) If $\nu$ is such that there exists an $f \in L_{1}(\mu, E)$ such that $|\nu|<|J(f)|$, then there is a $g \in L_{1}(\mu, E)$ with $J(g)=\nu$.

Proof. Clearly (c) and (d) are equivalent.

(a) $\Rightarrow$ (b). Assume that $E$ has order continuous norm. Let $\nu \in \mathrm{ca}_{\infty}(\mu, E)^{+}$be given, so there exists an $e \in E^{+}$such that $0<\nu(A)<\mu(A) e$ for all $A \in \Sigma$. Now 
define $T_{\nu}: L_{1}(\mu) \rightarrow E$ by setting

$$
T_{\nu}(f)=\int f d \nu
$$

for all $f \in L_{1}(\mu)$ (this can be done by first defining $T_{\nu}$ on the step functions and then extending it). It follows now that

$$
-e \leqslant T_{\nu}(f) \leqslant e
$$

for all $f \in L_{1}(\mu)$ with $\|f\|_{1}<1$. By [7, Theorem II.5.10] $T_{\text {, }}$ is weakly compact and hence, by [2, Theorem III.2.12], there exists an $f \in L_{\infty}(\mu, E)$ such that $J(f)=\nu$. Obviously $|f| \leqslant e \chi_{x}$ has to hold, so $f \in B_{\infty}(\mu, E)$. This shows that $J\left(B_{\infty}(\mu, E)\right)=$ $\mathrm{ca}_{\infty}(\mu, E)$.

(b) $\Rightarrow$ (a) Assume that $J\left(B_{\infty}(\mu, E)\right)=\mathrm{ca}_{\infty}(\mu, E)$. Then $B_{\infty}(\mu, E)$ is a Dedekind complete Riesz space since $\mathrm{ca}_{\infty}(\mu, E)$ is such. Hence $L_{1}(\mu, E)$ is Dedekind complete as well. By [1, p. 59] it follows that $E$ must have order continuous norm.

(a) $\&($ b) $\Rightarrow$ (d) Let $\nu \in m(\Sigma, E)$ be such that $0<\nu<J(f)$ for some $f \in$ $L_{1}(\mu, E)^{+}$. Clearly $\nu \in M(\mu, E)^{+}$has to hold. By Theorem 2.2(ii), there exists a sequence $\nu_{1}, \nu_{2}, \ldots$ in $\mathrm{ca}_{\infty}(\mu, E)^{+}$such that $\nu_{n} \uparrow \nu$. By assumption there exist $g_{1}, g_{2}, \ldots$ in $B_{\infty}(\mu, E)^{+}$such that $J\left(g_{n}\right)=\nu_{n}$ for all $n$. Obviously we have

$$
0 \leqslant g_{1} \leqslant g_{2} \leqslant \cdots<f \text {. }
$$

Again by [1, p. 59], $L_{1}(\mu, E)$ is Dedekind complete, so $g=\bigvee_{n=1}^{\infty} g_{n}$ exists in $L_{1}(\mu, E)$. It is immediate that $J(g)=\nu$. Finally, it is clear that (c) implies (b).

Next, we characterize the Banach lattices $E$ for which $J\left(L_{1}(\mu, E)\right)=M(\mu, E)$.

THEOREM 3.3. The following are equivalent.

(a) $J\left(L_{1}(\mu, E)\right)=M(\mu, E)=\operatorname{BCA}(\mu, E) \cap \mathrm{ca}_{1}(\mu, E)$.

(b) If $\left\{e_{n}: n \in \mathbf{N}\right\}$ is a norm bounded increasing sequence in $E^{+}$, then it has a norm limit.

(c) E does not contain a closed Riesz subspace isomorphic to $c_{0}$.

Proof. By [7, Theorem II.5.15], (b) and (c) are equivalent. Now, to show (a) $\Rightarrow(c)$, assume that $E$ contains $c_{0}$. Let $\nu: \Sigma \rightarrow c_{0}$ be the vector measure constructed by Lewis in [5]. It is shown in [5] that $\nu \in \operatorname{BCA}\left(\mu, c_{0}\right)$ and that $\nu \notin$ $J\left(L_{1}\left(\mu, c_{0}\right)\right)$. Furthermore, viewing $\nu$ as a measure into $l_{\infty}$, it is immediate that $\nu \in \mathrm{ca}_{\infty}\left(\mu, l_{\infty}\right)$. Hence $\nu \in \mathrm{ca}_{1}\left(\mu, c_{0}\right)$, so $\nu \in M\left(\mu, c_{0}\right)$. This contradicts the assumptions of (a).

Next, assume that (b) holds. Then $E$ has order continuous norm. Given $\nu \in$ $M(\mu, E)^{+}$, there exist $\nu_{1}, \nu_{2}, \ldots$ in $\mathrm{ca}_{\infty}(\mu, E)^{+}$such that $\nu_{n} \uparrow \nu$ (again by Theorem 2.2(ii)). By Theorem 3.3, there exist $g_{1}, g_{2}, \ldots$ in $B_{\infty}(\mu, E)^{+}$such that $J\left(g_{n}\right)=\nu_{n}$ for all $n$. Clearly, we have

$$
0 \leqslant g_{1}(\omega) \leqslant g_{2}(\omega)<\ldots
$$

$\mu$-a.e. on $\Omega$ (after choosing representants). Next, applying [2, Theorem II.1.4], we obtain

$$
\int\left\|g_{n}(\omega)\right\| d \mu(\omega)=\left\|\nu_{n}\right\|(\Omega)<\|\nu\|
$$


for all $n$, so $\left\{\left\|g_{n}(\omega)\right\|: n \in \mathbf{N}\right\}$ is bounded $\mu$-a.e. on $\Omega$. By assumption, there exists a $g: \Omega \rightarrow E^{+}$such that $\left\|g(\omega)-g_{n}(\omega)\right\| \rightarrow 0 \mu$-a.e. on $\Omega$. Since

$$
\int\|g(\omega)\| d \mu(\omega)=\lim \int\left\|g_{n}(\omega)\right\| d \mu(\omega)<\|\nu\|,
$$

it follows that $g \in L_{1}(\mu, E)^{+}$. It is immediate that $J(g)=\nu$ has to hold, which shows that (b) implies (a).

It remains to show that if $J\left(L_{1}(\mu, E)\right)=M(\mu, E)$, then

$$
M(\mu, E)=\operatorname{BCA}(\mu, E) \cap \operatorname{ca}_{1}(\mu, E) .
$$

Clearly $M(\mu, E)$ is contained in the intersection. For the converse direction, let $\nu \in \operatorname{BCA}(\mu, E) \cap \mathrm{ca}_{1}(\mu, E)$ be given. Clearly $|\nu| \in \mathrm{ca}_{1}(\mu)$. Furthermore, it is $\sigma-$ additive and $\mu$-absolutely continuous, so it remains to show that $|\nu|$ is of bounded variation. To this end, let $\left\{A_{1}, \ldots, A_{n}\right\}$ be a partition of $\Omega$. Fixing $i \in\{1, \ldots, n\}$, we have

$$
|\nu|\left(A_{i}\right)=\sup \left\{\sum_{j=1}^{k}\left|\nu\left(B_{j}\right)\right|: B_{1}, \ldots, B_{k} \text { a partition of } A_{i}\right\} .
$$

Since (b) holds it follows that

$$
\begin{aligned}
\left\||\nu|\left(A_{i}\right)\right\| & =\sup \left\{\left\|\sum_{j=1}^{k}\left|\nu\left(B_{j}\right)\right|\right\|: B_{1}, \ldots, B_{k} \text { a partition of } A_{i}\right\} \\
& \leqslant \sup \left\{\sum_{j=1}^{k}\left\|\nu\left(B_{j}\right)\right\|: B_{1}, \ldots, B_{k} \text { a partition of } A_{i}\right\}=\|\nu\|\left(A_{i}\right) .
\end{aligned}
$$

Hence

$$
\||\nu|\|=\sup \left\{\sum_{i=1}^{n}\left\||\nu|\left(A_{i}\right)\right\|: A_{1}, \ldots, A_{n} \text { a partition of } \Omega\right\}<\|\nu\| .
$$

The inverse inequality is obvious, so $\||\nu|\|=\|\nu\|$ and in particular $|\nu| \in$ $\operatorname{BCA}(\mu, E)$.

REMARKS. 1. A classical result due to Grothendieck describes the class of vector measures with values in an $L_{1}$ space having a $R N$-derivative (see [2, pp. 258, 259]). As $c_{0}$ is not contained in an $L_{1}$ space, Theorem 3.3 recovers Grothendieck's result.

2. Since a Banach space with RNP cannot contain $c_{0}$, we see immediately from Theorem 3.3 that the following are equivalent (compare also [4, Theorem 2]).

(a) $E$ has RNP.

(b) $E$ does not contain $c_{0}$ as a closed sublattice and for all $\nu \in \operatorname{BCA}(\mu, E)$ we have $\nu \lll \mu$.

3. Let $\nu \in \operatorname{ob}(\Sigma, E)$ be given. At a first glance it is not clear how the classical notion of $\mu$-absolute continuity $(\nu \ll \mu)$ for $\nu$ and the notion $\nu \lll \mu$ are related. Clearly, if $E$ has order continuous norm, then $\nu \lll \mu$ implies $\nu \ll \mu$. Conversely, taking for instance $E=L_{1}(\mu)$, we see from Remark 2 above that $\nu \ll \mu$ does not necessarily imply $\nu \lll \mu$, not even if $E$ has order continuous norm. If $E$ does not have order continuous norm, the notions $\nu \ll \mu$ and $\nu \lll \mu$ do not seem to be related at all. Therefore, the following is of interest. First recall that an element 
$e \in E^{+}, e \neq 0$ is called an atom if it follows from $0<f<e$ that $f=\alpha e$ for some $\alpha \in \mathbf{R}^{+}$. Furthermore, $E$ is called purely atomic if every $f \in E^{+}, f \neq 0$ dominates an atom of $E$.

LEMMA 3.4. Assume that $E$ is purely atomic and that $E$ has order continuous norm. Let $\nu \in \mathrm{ob}(\Sigma, E)$ be given. Then $\nu \ll \mu$ if and only if $\nu \lll \mu$.

Proof. We may assume that $\nu \geqslant 0$. It suffices to show that $\nu \ll \mu$ implies $\nu \lll \mu$. So assume that $\nu \ll \mu$ and that $\nu \notin \mathrm{ca}_{1}(\mu, E)$. Then there exists an atom $e \in E^{+}$ such that

$$
\bigwedge_{\varepsilon>0} \bigvee_{\mu(A)<\varepsilon} \nu(A)>e .
$$

Since $e$ is an atom, it follows that for all $n \in \mathbf{N}$, there exists an $A_{n} \in \Sigma$ such that $\mu\left(A_{n}\right)<n^{-1}$ and $\nu\left(A_{n}\right)>e$. In particular we have

$$
\left\|\nu\left(A_{n}\right)\right\|>\|e\|>0
$$

contradicting $\nu \ll \mu$.

Combining the above lemma with Remark 2, we obtain

COROllary 3.5. Assume that $E$ is purely atomic. Then the following are equivalent

(a) $E$ has RNP.

(b) $E$ does not contain $c_{0}$ as a closed sublattice.

\section{REFERENCES}

1. D. I. Cartwright, The order completeness of some spaces of vector valued functions, Bull. Austral. Math. Soc. 11 (1974), 57-61.

2. J. Diestel and J. J. Uhl, Jr., Vector measures, Math. Surveys, no. 15, Amer. Math. Soc., Providence, R. I., 1977.

3. B. Faires and T. J. Morrison, The Jordan decompasition of vector valued measures, Proc. Amer. Math. Soc. 60 (1976), 139-143.

4. N. Ghoussoub and M. Talagrand, Order dentability and the Radon-Nikodym Property in Banach lattices, Math. Ann. 243 (1979), 217-226.

5. D. R. Lewis, A vector measure with no derivative, Proc. Amer. Math. Soc. 32 (1972), 535-536.

6. H. P. Lotz, The Radon-Nikodym Property in Banach lattices (to appear).

7. H. H. Schaefer, Banach lattices and positive operators, Springer-Verlag, Berlin and New York, 1974.

Mathematisch Institut der Ruksuniversiteit, Leiden, The NetherLands

Current address: KSEPL, L6-206, Volmer Laan 6, Rÿswÿk (Z. H.), The Netherlands 